\title{
SURFACES OF VERTICAL ORDER 3 ARE TAME
}

\author{
BY R. A. JENSEN AND L. D. LOVELAND ${ }^{1}$
}

Communicated by Orville Harrold, July 17, 1969

We define a 2 -sphere $S$ in $E^{3}$ to have vertical order $n$ if each vertical line intersects $S$ in no more than $n$ points. The main result in this paper is the following

THEOREM 1. If $S$ is a 2-sphere in $E^{3}$ having vertical order 3 , then $S$ is tame.

This is the best theorem possible in the sense that examples are known of wild 2-spheres in $E^{3}$ having vertical order 4 [5]. In Theorem 2 to follow we generalize Theorem 1 to compact 2-manifolds in $E^{3}$.

Previous work concerned with the nature of the intersection of vertical lines with a 2 -sphere in $E^{3}$ has been done by Bing [1, Theorem 7.3]; [3].

Proof of Theorem 1 . The vertical line in $E^{3}$ containing the point $x$ is denoted by $L_{x}$, and we refer to the bounded component of $E^{3}-S$ as Int $S$. If $x \in$ Int $S$ it is easy to see that $L_{x} \cap S$ consists of two points. In this case the point with largest third coordinate is denoted by $U_{x}$ and the other by $V_{x}$. Welet $U=\left\{U_{x} \mid x \in \operatorname{Int} S\right\}$ and $V=\left\{V_{x} \mid x \in \operatorname{Int} S\right\}$, and we note that $U$ and $V$ are both open subsets of $S$. A bicollar can be constructed for a neighborhood of each point of $U \cup V$ using short vertical intervals. Thus $S$ is locally tame at each point of $U \cup V$ [2].

Let $R=S-(U \cup V)$. The proof that $S$ is tame is completed by showing that $R$ is a tame simple closed curve, since a 2 -sphere that is locally tame modulo a tame simple closed curve is known to be tame [4].

It will follow that $R$ is a simple closed curve once we show that each of $U$ and $V$ is connected and that each point $p \in R$ is arcwise accessible from both $U$ and $V[7$, p. 233]. Let $\theta$ be an arc in Int $S$ $\cup\{p\}$ such that $p$ is an endpoint of $\theta$. We now show that the vertical projection $\sigma$ of $\theta$ into $U \cup\{p\}$ is continuous. To accomplish this we take a sequence $\left\{x_{i}\right\}$ of points in $\theta$ converging to $x_{0}$ and we prove that the sequence $\left\{\sigma\left(x_{i}\right)\right\}$ converges to $\sigma\left(x_{0}\right)$. Let $L_{i}(i=0,1,2, \cdots)$ be the vertical interval from $x_{i}$ to $\sigma\left(x_{i}\right)$ (if $x_{i}=p$, then $L_{i}$ is degenerate),

\footnotetext{
AMS Subject Classifications. Primary 5705; Secondary 5478.

Key Words and Phrases. Tame 2-spheres, tame surfaces, surfaces in $E^{\mathbf{3}}$.

1 This research was partially supported by the National Science Foundation under GP-8454.
} 
and let $L=$ limit superior $\left\{L_{i}\right\}$. Then $L$ is an interval (possibly degenerate) in $L_{x_{0}}$ having lower endpoint $x_{0}$. The upper endpoint of $L$ must lie in $S$ so it cannot lie below $\sigma\left(x_{0}\right)$. On the other hand $L$ cannot properly contain $L_{0}$ either, since then there would be limit points of Int $S$ in Ext $S$. Thus $L=L_{0}$, and we see that $\left\{\sigma\left(x_{i}\right)\right\}$ converges to $\sigma\left(x_{0}\right)$. Since $\sigma(\theta)$ is the continuous image of an arc, it must contain an arc having $p$ as an endpoint. Now $p$ is arcwise accessible from $V$ by the same reasoning. A similar argument would establish the arcwise connectivity of both $U$ and $V$, so we may conclude that $R$ is a simple closed curve.

All that remains is to show that $R$ is tame. We let $p \in R$ and we let $\epsilon>0$. In the remainder of the proof we construct a 2 -sphere $S^{\prime}$ such that $p \in \operatorname{Int} S^{\prime}, S^{\prime}$ has diameter less than $\epsilon$, and $S^{\prime} \cap R$ consists of two points. This is enough to ensure the tameness of $R$ since it implies that $R$ satisfies property $P$ of [6].

Let $N$ be a round open neighborhood of $p$ with diameter less than $\epsilon$, and let $\alpha$ and $\beta$ be two arcs in $R$ such that $\alpha \cap \beta=\{p\}, \alpha \cup \beta \subset N$, and no point of $N \cap R$ lies vertically above $p$. There is an arc $\gamma$ joining the two endpoints of $\alpha \cup \beta$ such that Int $\gamma \subset U$ and $\alpha \cup \beta \cup \gamma$ is a simple closed curve $J$ bounding an open disk $W$ in $U$. Since the vertical projection $\pi$ of $E^{3}$ onto a horizontal plane $E^{2}$ is continuous, there exist $\operatorname{arcs} \alpha^{\prime}$ and $\beta^{\prime}$ in $\pi(\alpha)$ and $\pi(\beta)$, respectively, such that $\pi(\mathrm{Bd} \alpha)=\mathrm{Bd} \alpha^{\prime}$ and $\pi(\mathrm{Bd} \beta)=\operatorname{Bd} \beta^{\prime}$. The proof is completed in two cases.

Case 1. $\alpha^{\prime} \cap \beta^{\prime}$ is nondegenerate. In this case we let $x^{\prime}$ be a point of $\alpha^{\prime} \cap \beta^{\prime}$ such that $x^{\prime} \neq \pi(p)=p^{\prime}$, and we let $x \in \alpha$ and $y \in \beta$ be two points such that $\pi(x)=\pi(y)=x^{\prime}$. Let $f$ be an arc from $x$ to $y$ such that $f-\{x, y\} \subset W$, and notice that $f^{\prime}=\pi(f)$ is a simple closed curve. It is not difficult to show that $p$ lies in Int $H$ where $H=\pi^{-1}\left(f^{\prime}\right)$ and Int $H$ is the component of $E^{3}-H$ whose intersection with $E^{2}$ is bounded. We form a 2-sphere $T$ in $\bar{N}$ by taking the union of $H \cap N$ with the two disks in $(\operatorname{Bd} N) \cap(H \cup$ Int $H)$. If $T \cap R=\{x, y\}$ we let $T=S^{\prime}$. Otherwise $T \cap R$ consists of three points and it follows that $R$ cannot pierce $T$ at all three points. Thus $R$ must be tangent to $H$ at one point, and we may move $T$ slightly to the nontangency side of $H$ near the nonpiercing point to form $S^{\prime}$ in this case.

Case 2. $\alpha^{\prime} \cap \beta^{\prime}=\left\{p^{\prime}\right\}$. Let $\gamma^{\prime}=\pi(\gamma)$ and $W^{\prime}=\pi(W)$. In this case $\alpha^{\prime} \cup \beta^{\prime} \cup \gamma^{\prime}$ is a simple closed curve $J^{\prime}$ bounding a disk $D^{\prime}$ in $E^{2}$. It is not difficult to see that $W^{\prime} \subset$ Int $D^{\prime}$ because $W^{\prime} \cap J^{\prime}=\varnothing$ and $W^{\prime}$ is arcwise connected. Let $N_{1}$ and $N_{2}$ be round open neighborhoods of $p$ such that $\bar{N}_{1} \cap R \subset \alpha \cup \beta$ and each pair of points of $R \cap N_{2}$ lies in an arc in $(\alpha \cup \beta) \cap N_{1}$. If $x^{\prime} \in \alpha^{\prime} \cap \pi\left(N_{2}\right)$ and $y^{\prime} \in \beta^{\prime} \cap \pi\left(N_{2}\right)$ there is an arc $g^{\prime}$ from $x^{\prime}$ to $y^{\prime}$ such that $g^{\prime}-\left\{x^{\prime}, y^{\prime}\right\} \subset E^{2}-D^{\prime}$ and $g^{\prime} \subset \pi\left(N_{1}\right)$. 
The idea of the remainder of the proof is to obtain an arc $f^{\prime}$ from $x^{\prime}$ to $y^{\prime}$ such that $f^{\prime}-\left\{x^{\prime}, y^{\prime}\right\} \subset \pi\left(N_{1}\right) \cap W^{\prime}$, and then to construct the 2-sphere $S^{\prime}$ using part of the infinite vertical cylinder $H=\pi^{-1}\left(f^{\prime} \cup g^{\prime}\right)$ and two disks in Bd $N_{1}$. Of course, this requires a nice enough selection of $x^{\prime}$ and $y^{\prime}$ to insure that $H$ intersects $R$ in a controlled manner.

Suppose we are able to select $x^{\prime} \in \alpha^{\prime}$ and $y^{\prime} \in \beta^{\prime}$ each having exactly one point, say $x$ and $y$ respectively, of $\alpha \cup \beta$ vertically above it. Then an $\operatorname{arc} f$ can be constructed in $N_{1} \cap(W \cup\{x, y\})$ whose projection $\pi(f)$ satisfies the desired conditions on $f^{\prime}$, and it would follow that $H \cap(\alpha \cup \beta)=\{x, y\}$. Thus $S^{\prime}$ could be chosen as the boundary of the 3-cell ( $H \cup$ Int $H$ ) $\cap \bar{N}_{1}$, and it would follow that $R \cap S^{\prime}=\{x, y\}$. We show now that such points $x^{\prime}$ and $y^{\prime}$ can always be found.

Suppose that for each $x^{\prime} \in \beta^{\prime}$ the set $\pi^{-1}\left(x^{\prime}\right) \cap \beta$ contains at least two points. We can select $x^{\prime} \in \beta^{\prime}$ such that $\pi^{-1}\left(x^{\prime}\right) \cap \beta$ contains two points $x_{1}$ and $x_{2}$ having the property that every open arc in $\beta$ with either $x_{1}$ or $x_{2}$ as an endpoint intersects $\pi^{-1}\left(\beta^{\prime}\right)$. This is possible because $\beta-\pi^{-1}\left(\alpha^{\prime} \cup \beta^{\prime}\right)$ has at most countably many components, and any point not in the projection of their endpoints would satisfy the conditions on $x^{\prime}$.

We choose disjoint arcs $A_{1}$ and $A_{2}$ such that $x_{i} \in \operatorname{Int} A_{i} \subset A_{i} \subset \beta$ and $A_{i} \cap L_{x_{i}}=\left\{x_{i}\right\} \quad(i=1,2)$, and we choose disjoint disks $D_{1}$ and $D_{2}$ such that $A_{i} \subset \operatorname{Bd} D_{i},\left(D_{i}-A_{i}\right) \subset W$, and $\operatorname{Bd} D_{i}-A_{i}$ is an open arc $B_{i}$ in $W(i=1,2)$. In view of the selection of $x^{\prime}$ we may assume that the endpoints of each $A_{i}$ lie in $\pi^{-1}\left(\beta^{\prime}\right)$. This implies that the open arcs $B_{i}^{\prime}=\pi\left(B_{i}\right)$ have their endpoints in $\beta^{\prime}$. Notice that $B_{1}^{\prime} \cap B_{2}^{\prime}=\varnothing$, for otherwise a vertical line through a point of $B_{1}^{\prime} \cap B_{2}^{\prime}$ would intersect $W$ twice; and recall that $\pi\left(D_{1} \cup D_{2}\right) \subset \pi(W \cup J) \subset D^{\prime}$. Since $x^{\prime}$ lies in the boundary of both $\pi\left(D_{1}\right)$ and $\pi\left(D_{2}\right)$, we see that the endpoints of each $B_{i}^{\prime}$ separate $x^{\prime}$ from $\gamma^{\prime}$ in $\mathrm{Bd} D^{\prime}$. Thus the closure of one of $B_{1}^{\prime}$ and $B_{2}^{\prime}$, say $B_{1}^{\prime}$, separates the other from $\gamma$ in $D^{\prime}$. This forces $\bar{B}_{2}^{\prime}$ to separate $B_{1}^{\prime}$ from $x^{\prime}$ in $D^{\prime}$, and yields a contradiction since there is an $\operatorname{arc}$ in $D_{1}$ from $x_{1}$ to a point of $B_{1}$ missing $B_{2}$.

THEOREM 2. If $S$ is a compact 2-manifold in $E^{3}$ having vertical order 3 , then $S$ is tame.

We restrict ourselves here to an outline of the proof of Theorem 2. By working with a component of $S$ we may suppose that $S$ is connected and consequently that $S$ has exactly two complementary domains. The sets $U, V$, and $R$ are defined just as in the proof for Theorem 1, and in the same way we see that $U$ and $V$ are connected, open, and locally tame. In this case $R$ is a finite collection of disjoint simple closed curves each of which can be proven tame by establishing 
Properties $P$ and $Q$ of [6] as before. Thus $S$ is tame, since it is locally tame modulo a finite collection of tame simple closed curves.

\section{REFERENCES}

1. R. H. Bing, Tame Cantor sets in $E^{3}$, Pacific J. Math. 11 (1961), 435-446. MR 24 \#A539.

2. - A surface is tame if its complement is 1-ULC, Trans. Amer. Math. Soc. 101 (1961), 294-305. MR 24 \#A1117.

3. - Improving the intersections of lines and surfaces, Michigan Math. J. 14 (1967), 155-159. MR 34 \#6743.

4. P. H. Doyle and J. G. Hocking, Some results on tame disks and spheres in $E^{3}$, Proc. Amer. Math. Soc. 11 (1960), 832-836. MR 23 \#A4133.

5. R. H. Fox and E. Artin, Some wild cells and spheres in three-dimensional space, Ann. of Math. (2) 49 (1948), 979-990. MR 10, 317.

6. O. G. Harrold, Jr., H. C. Griffith and E. E. Posey, A characterization of tame curves in 3-space, Trans. Amer. Math. Soc. 79 (1955), 12-34. MR 19, 972.

7. R. L. Moore, Foundations of point set theory, Amer. Math. Soc. Colloq. Publ., vol. 32, Amer. Math. Soc., Providence, R. I., 1949.

University of Miami, Coral Gables, Florida 33124 and

Utah State University, Logan, Utah 84321 